\title{
On $\boldsymbol{F}$-connections and associated non- linear connections
}

\author{
Dedicated to Professor Dr. W. Barthel, \\ wishing a quick recovery of his health
}

By

Makoto Matsumoto

(Received August 23, 1968)

In 1963, W. Barthel [1] developed an elegant theory of holonomy groups of homogeneous non-linear connections. He defined a homogeneous non-linear connection on a differentiable manifold $M$ as a special distribution on the tangent bundle $T(M)$.

As is well-known (for example, see [9]), a linear connection on $M$, however, can be defined as a connection in the bundle of linear frames $L(M)$ over $M$, and then its holonomy group is a subgroup of $G L(n, R)$ acting on $L(M)$.

The purpose of the present paper is to give a concept of an $F$ connection, a collection of special distributions on $L(M)$, and to show that any homogeneous non-linear connection in $T(M)$ is associated with an F-connection. For this purpose, a concept of Finsler connections will be quite useful. The first section is devoted to summarize basic concepts of Finsler connections, which have been described in a series of our papers $[2], \cdots,[8]$. In the second section, some properties of homogeneous Finsler connections will be derived. Then, the main result will be given in Theorem 6 of the third section. 


\section{§1. Introduction}

This section is an introductory summary of basic concepts of Finsler connections, zeeded for the later treatment. Throughout the present paper, we denote by $P_{p}$ the tangent space to a differentiable manifold $P$ at a point $p$, and by $B^{v}$ the vertical distribution $b \in B$ $\rightarrow B_{b}^{v}$ on the total space $B$ of a fibre bundle, where $B_{b}^{v}$ is the vertical subspace of the tangent space $B_{b}$, the kernel of the differential of the projection of $B$. It is further noted that the differential of a differentiable mapping $\mu$ will be denoted by $\mu$ itself.

[1] We shall consider a differentiable $n$-manifold $M$ and the following fibre bundles.

The bundle of non-zero tangent vectors $T(M)(M, \tau, F, G)$ :

$M \cdots \cdots$ base space, $\quad \tau \cdots \cdots$ projection $T(M) \rightarrow M$,

$F \cdots \cdots$ standard fibre (real vector $n$-space),

$G=G L(n, R) \cdots \cdots$ structural group.

The principal bundle of linear frames $L(M)(M, \pi, G)$ :

$M \cdots \cdots$ base space, $\quad \pi \cdots \cdots$ projection $L(M) \rightarrow M$,

$G=G L(n, R) \cdots \cdots$ structural group.

The induced bundle $\tau^{-1} L(M)=F(M)\left(T(M), \pi_{1}, G\right)$ :

$F(M)=\{(y, z) \in T(M) \times L(M) \mid \tau y=\pi z\} \cdots \cdots$ total space,

$T(M) \cdots \cdots$ base space,

$\pi_{1} \cdots \cdots$ projection $F(M) \rightarrow T(M), \quad[(y, z) \rightarrow y]$,

$G=G L(n, R) \cdots \cdots$ structural group.

The bundle $F(M)$ is called the Finsler bundle of $M$. The operation $r$ of $G$ on $F$ is determined by

$$
r: G \times F \rightarrow F, \quad\left[\left(g=\left(g_{b}^{a}\right), f=f^{a} e_{a}\right) \rightarrow g f=g_{b}^{a} f^{b} e_{a}\right],
$$

with respect to a fixed base $\left(e_{a}\right), a=1, \cdots, n$, of $F$. Next, the operation $t$ of $G$ on the total space $L(M)$ is given by

$$
t: L(M) \times G \rightarrow L(M), \quad\left[\left(z=\left(z_{a}\right), g=\left(g_{b}^{a}\right)\right) \rightarrow z g=\left(z_{b} g_{a}^{b}\right)\right],
$$

and then, the operation $T$ of $G$ on the total space $F(M)$ is induced 
from $t$ as follows.

$T: F(M) \times G \rightarrow F(M),[((y, z), g) \rightarrow(y, z g)]$.

Let us denote by $R^{+}$the differentiable manifold composed of all the positive numbers, and let $h$ be a mapping

$$
h: R^{+} \times T(M) \rightarrow T(M), \quad[(\alpha, y) \rightarrow \alpha y] .
$$

Then, the mapping

$$
H: R^{+} \times F(M) \rightarrow F(M), \quad[(\alpha, u=(y, z)) \rightarrow \alpha u=(\alpha y, z)]
$$

is induced from $h$. A transformation ${ }_{\alpha} h$ of $T(M)$ is obtained from the above $h$ by becoming $\alpha \in R^{+}$fixed. Then, a distribution $D: y$ $\in T(M) \rightarrow D_{y} \subset T(M)_{y}$ on $T(M)$ is called $h$-invariant, if ${ }_{\alpha} h D_{y}=D_{\alpha y}$ holds good at any $y$ and for any $\alpha$. The notion of the $H$-invariance will be similarly defined for distributions on $F(M)$.

The Finsler subbundle $F(x)$ at a point $x \in M$ is by definition a subbundle of $F(M)$ over a fibre $\tau^{-1} x \subset T(M)$. It will be obvious that the tangent space $F(x)_{u}$ is the subspace of $F(M)_{u}$ given by $F(M)_{u}^{a}=\left\{X \in F(M)_{u} \mid \tau \cdot \pi_{1} X=0\right\}$, which is called the quasi vertical subspace of $F(M)_{u}$.

[2] We shall present here concepts of some connections in $T(M)$, $L(M)$ and $F(M)$.

Definition 1. A distribution $N: y \in T(M) \rightarrow N_{y} \subset T(M)$, on $T(M)$ is called a non-linear connection in $T(M)$, if $N$ is a complement of the vertical distribution $T^{v}$, that is,

$$
T(M)_{y}=N_{y} \oplus T_{y}^{v}, \quad \text { (direct sum), }
$$

at any point $y \in T(M)$. Further, $N$ is called homogeneous, if $N$ is $h$-invariant.

Definition 2. An F-connection $\Gamma_{F}$ in $L(M)$ is a collection $\left\{\Gamma_{(f)}\right\}$ of distributions $\Gamma_{(f)}: z \in L(M) \rightarrow \Gamma_{(f) z} \subset L(M)_{z}$, corresponding to any $f \in F$, which satisfies 
(1) $L(M)_{z}=\Gamma_{(f) z} \oplus L_{z}^{v}$, at any point $z \in L(M)$,

(2) $t_{g} \Gamma_{(f) z}=\Gamma_{\left(g^{-1} f\right) z}$, at any point $z$ and for any $g \in G$.

The above mapping $t_{g}$ is a right translation of $L(M)$ by $g \in G$, which is obtained from $t$ by becoming $g$ fixed. It is remarked that each $\Gamma_{(f)}$ is not a connection in $L(M)$ in the ordinary sense, because (2) differs a little from the $t$-invariance of an ordinary connection. As for a connection $\Gamma$ in $L(M)$, the associated connection $\Gamma^{*}$ will be obtained in $T(M)$. In fact, the total space $T(M)$ is identified with the quotient space $(L(M) \times F) / G$ by the operation $(z, f) \in L(M) \times F \rightarrow\left(z g, g^{-1} f\right), g \in G$, and hence the canonical projection $L(M) \times F \rightarrow(L(M) \times F) / G$ gives

$$
a: L(M) \times F \rightarrow T(M),[(z, f) \rightarrow z f],
$$

where we denote by $z f$ the equivalence class containing $(z, f)$. The mapping $a_{f}: L(M) \rightarrow T(M)$ obtained from $a$ by becoming $f \in F$ fixed is called the associated mapping. Then, the associated connection $\Gamma^{*}$ is defined by $\Gamma_{y}^{*}=a_{f} \Gamma_{z}, y=z f$. In the same way, a non-linear connection $N$ will be obtained from an $F$-connection $\Gamma_{F}$ as follows.

Proposition 1. Let $\Gamma_{F}=\left\{\Gamma_{(f)}\right\}$ be an F-connection in $L(M)$, and then by the equation

$$
N_{y}=a_{f} \Gamma_{(f) z}, \quad y=z f,
$$

a distribution $N: y \in T(M) \rightarrow N$, is well defined. Then $N$ is a non-linear connection in $T(M)$.

The proof is omitted, because it will be easily obtained. The non-linear connection $N$ as above introduced is called the associated non-linear connection with $\Gamma_{F}$.

From now on, we shall treat the Finsler bundle $F(M)$ of $M$, and first give the following definition.

Definition 3. A vertical connection $\Gamma^{v}$ in $F(M)$ is a distribu 
tion $u \in F(M) \rightarrow \Gamma_{u}^{v} \subset F(M)_{u}$, such that the restriction $\Gamma^{v} \mid F(x)$ of $\Gamma^{v}$ to each Finsler subbundle $F(x)$ is a connection in $F(x)$.

Therefore, $\Gamma^{v}$ is a vertical connection, if the following conditions be satisfied:

(1) $F(M)_{u}^{o}=\Gamma_{u}^{v} \oplus F_{u}^{v}$, at any point $u=F(M)$,

(2) $T$-invariant: $T_{g} \Gamma_{u}^{v}=\Gamma_{u_{g}}^{v}$, for any $g \in G$ and at any $u$ $\in F(M)$.

The above mapping $T_{g}$ is a right translation of $F(M)$ by $g$, which is obtained from $T$ by becoming $g \in G$ fixed. We shall give a differentiable base $\left(B^{v}\left(e_{a}\right)\right), a=1, \cdots, n$, of the vertical connection $\Gamma^{v}$. For this purpose, we shall first introduce a parallel vector field $P(f)$ on $F$, corresponding to $f \in F . \quad P(f)$ is induced from a 1parameter $(t)$ group of transformations $\left\{s_{t f}\right\}$ of $F$, where the mapping $s_{f}, f \in F$, is the summation $f_{1} \in F \rightarrow f_{1}+f$. Then, a v-basic vector field $B^{\circ}(f)$ on $F(M)$, corresponding to $f \in F$, is defined by

$$
B^{v}(f)_{u}=l_{u}^{v} \cdot z\left(P(f)_{\gamma(u)}\right),
$$

at a point $u=(y, z)$, where $l_{u}^{v}$ is the lift to $u$ with respect to $\Gamma^{v}, z$ the differential of the admissible mapping ${ }_{2} a: F \rightarrow T(M)$ obtained from the mapping $a$ by becoming a frame $z$ fixed, and $r$ is the characteristic field $u=(y, z) \in F(M) \rightarrow z^{-1} y=\left({ }_{2} a\right)^{-1} y$ [2, p. 3]. It will be obvious that $n v$-basic vector fields $B^{v}\left(e_{a}\right), a=1, \cdots, n$, give a base of $\Gamma^{v}$ at every point of $F(M)$.

Next, we shall introduce a special vertical connection $F^{i}$. Since $F(M)$ is the induced bundle $\tau^{-1} L(M)$, there is the induced mapping $\pi_{2}: F(M) \rightarrow L(M),[(y, z) \rightarrow z]$. The characteristic field $\gamma$, together with the induced mapping $\pi_{2}$, gives the diffeomorphism

$$
i=\left(\pi_{2}, \gamma\right): F(M) \rightarrow L(M) \times F, \quad\left[(y, z) \rightarrow\left(z, z^{-1} y\right)\right],
$$

and its inverse $i^{-1}$ is

$$
i^{-1}: L(M) \times F \rightarrow F(M), \quad[(z, f) \rightarrow(z f, z)] .
$$

By means of this identification $i$, a parallel vector field $P(f)$ on $F$, 
corresponding to $f \in F$, gives a vector field $Y(f)=i^{-1}(0, P(f))$ on $F(M)$, which is called the induced fundamental vector field, corresponding to $f$. It is obvious that any $Y(f)$ is contained in the induced vertical subspace $F_{u}^{i}=\left\{X \in F(M)_{u} \mid \pi_{2} X=0\right\}$ of $F(M)_{u}$.

Proposition 2. The induced vertical distribution $F^{i}: u \in F(M)$ $\rightarrow F_{u}^{i}$ on $F(M)$ is a vertical connection, and the v-basic vector field $B^{v}(f)$ with respect to $F^{i}$ is nothing but the above $Y(f)$.

The proof is omitted, kecause it will be easily obtained. It is remarked that $Y(f)$ is induced from the 1-parameter $(t)$ group of transformations $\left\{S_{t f}\right\}$ of $F(M)$, where $S_{f}=i^{-1} \cdot\left(1, s_{f}\right) \cdot i$. Since the equation $\left[Y\left(f_{1}\right), Y\left(f_{2}\right)\right]=0, f_{1}, f_{2} \in F$, will be derived in virtue of the identification $i$, the vertical connection $F^{i}$ as above obtained should be called flat.

[3] We are now in a pcsition to intrcduce a concept of Finsler connections.

Definition 4. A Finsler connection $(\Gamma, N)$ of $M$ is a pair of a connection $\Gamma$ in $F(M)$ and a non-linear connection $N$ in $T(M)$.

Given a Finsler connection $(\Gamma, N)$, we obtain the distribution $\Gamma^{*}$, defined by the equation

$$
\Gamma_{u}^{v}=l_{u} T_{y}^{v}, \quad \text { at a point } u,
$$

where $y=\pi_{1} u \in T(M)$, and $l_{u}$ is the lift to $u$ with respect to the connection $\Gamma$. It will be easy to show that the above $\Gamma^{r}$ is a vertical connection, which is called the subordinate vertical connection to $(I, N)$.

Definition 5. A Finsler pair $\left(\Gamma^{h}, \Gamma^{v}\right)$ in $F(M)$ is a pair of two distributions $\Gamma^{h}: u \in F(M) \rightarrow \Gamma_{u}^{h} \subset F(M)_{u}$ and $\Gamma^{v}: u \in F(M) \rightarrow \Gamma_{u}^{v}$ $\subset F(M)_{\text {u }}$, both on $F(M)$, which satisfies

(1) $\quad F(M)_{u}=\Gamma_{u}^{h} \oplus \Gamma_{u}^{v} \oplus F_{u}^{v}, \quad$ for any $u \in F(M)$, 
(2) both of $\Gamma^{h}$ and $\Gamma^{v}$ are $T$-invariant,

(3) $\pi_{1} \Gamma_{u}^{v}=T_{y}^{v}, \quad y=\pi_{1} u, \quad$ for any $u \in F(M)$.

It is clear that the second distribution $\Gamma^{v}$ of a Finsler pair $\left(\Gamma^{h}, \Gamma^{v}\right)$ is a vertical connection in $F(M)$.

The following theorem mears that a Finsler connection can be also defined as a Finsler pair.

Theorem 1. There is a natural one-to-one correspondence between the set of Finsler connections of $M$ and the set of Finsler pairs in $F(M)$.

As will be easily verified, the correspondence $(\Gamma, N) \rightarrow\left(\Gamma^{h}, \Gamma^{v}\right)$ is given by

$$
\begin{aligned}
& \Gamma_{u}^{h}=l_{u} N_{y}, \quad y=\pi_{1} u, \\
& \Gamma^{v} \cdots \cdots \text { subordinate vertical connection, }
\end{aligned}
$$

while the inverse correspondence $\left(\Gamma^{h}, \Gamma^{v}\right) \rightarrow(\Gamma, N)$ is

$$
\begin{aligned}
& \Gamma_{u}=\Gamma_{u}^{h} \oplus \Gamma_{u}^{v}, \\
& N_{y}=\pi_{1} \Gamma_{u}^{h}, \quad u \in \pi_{1}^{-1} y .
\end{aligned}
$$

In the following, we shall often express $(\Gamma, N)=\left(\Gamma^{h}, \Gamma^{v}\right)$, when $(\Gamma, N)$ and $\left(\Gamma^{h}, \Gamma^{v}\right)$ correspond each other by the above rule.

We shall give a differentiable base $\left(B^{n}\left(e_{a}\right)\right), a=1, \cdots, n$, of the distribution $\Gamma^{h}$. In order to do this, we first introduce an $h$-basic vector field $B^{h}(f)$, corresponding to $f \in F$, by the equation

$$
B^{k}(f)_{u}=l_{u} \cdot l_{y}(z f),
$$

at a point $u=(y, z)$, where $l_{u}$ and $l_{y}$ are the respective lifts with respect to $\Gamma$ and $N$. It then follows that $n h$-basic vector fields $B^{h}\left(e_{a}\right), a=1, \cdots, n$, give a base of $\Gamma^{h}$. As a consequence, $2 n$ vector fields $B^{h}\left(e_{a}\right), B^{v}\left(e_{a}\right), a=1, \cdots, n$, give a base of the connection $\Gamma$.

Let us project a Finsler pair $\left(\Gamma^{h}, \Gamma^{v}\right)$ on the bundle of linear frames $L(M)$ by means of the induced mapping $\pi_{2}: F(M) \rightarrow L(M)$. 
Then, corresponding to any $f \in F$, we obtain two distributions $\Gamma_{(f)}$ and $\Gamma_{(f)}^{v}$ on $L(M)$, such that

$$
\Gamma_{(f) z}=\pi_{2} \Gamma_{u}^{h}, \quad \Gamma_{(f) z}^{v}=\pi_{2} \Gamma_{u}^{v}, \quad u=i^{-1}(z, f) .
$$

We are not interested in the latter $\Gamma_{(f)}^{v}$, because it is vertical, that is, contained in the vertical distribution $L^{v}$. On the other hand, the former $\Gamma_{(f)}$ is very important, because it constitutes an $F$-connection $\Gamma_{F}=\left\{\Gamma_{(f)}\right\}$, as will be easily shown. This $\Gamma_{F}$ is called the subordinate $F$-connection to the Finsler connection $(\Gamma, N)=\left(\Gamma^{h}, \Gamma^{v}\right)$.

Definition 6. A Finsler triad $\left(\Gamma_{F}, N, \Gamma^{v}\right)$ of $M$ is a triad of an $F$-connection $\Gamma_{F}$ in $L(M)$, a non-linear connection $N$ in $T(M)$, and a vertical connection $\Gamma^{v}$ in $F(M)$.

Then, the following theorem means that a Finsler connection can be thought of as a Finsler triad.

Theorem 2. There is a natural one-to-one correspondence between the set of Finsler connections of $M$ and the set of Finsler triads on $M$.

The correspondence $(\Gamma, N)=\left(\Gamma^{h}, \Gamma^{v}\right) \rightarrow\left(\Gamma_{F}, N, \Gamma^{v}\right)$ is given by $\Gamma_{F} \cdots \cdots$ subordinate $F$-connection, while the inverse correspondence $\left(\Gamma_{F}, N, \Gamma^{v}\right) \rightarrow(\Gamma, N)=\left(\Gamma^{h}, \Gamma^{v}\right)$ is

$$
\Gamma_{u}^{h}=\left\{X \in F(M)_{u} \mid \pi_{1} X \in N_{y}, \pi_{2} X \in \Gamma_{(f)}, \quad y=\pi_{1} u, f=\gamma(u)\right\} .
$$

[4] We shall give a modern definition of tensor field appearing in the classical theory of Finsler spaces, whose components are functions not only of point, but also of element of support. Let $V$ be a vector space and $\rho: G \rightarrow G L(V)$ be a representation of $G$ $=G L(n, R)$ on $V$. Then, a Finsler tensor field $K$ of $\rho$-type is by definition a $V$-valued function on $F(M)$, satisfying the equation $K \cdot T_{g}=\rho\left(g^{-1}\right) K$ for any $g \in G$. If $V$ is the tensorial product 
$F_{s}^{r}=\underbrace{F \otimes \cdots \otimes}_{r} F_{s} \underbrace{*}_{s} \otimes \cdots \otimes F^{*}$ (space of linear mappings $\underbrace{F^{*} \times \cdots \times F^{*}}_{r}$ $\times \underbrace{F \times \cdots \times F}_{s} \rightarrow R)$ and $\rho$ is the usual representation, then $K$ is called of $(r, s) \cdot$ type.

For a typical example, the characteristic field $r$ is a Finsler tensor field of $(1,0)$-type. In order to show another example, we shall consider the difference between a general vertical connection $\Gamma^{v}$ and the vertical flat connection $F^{i}$. Then, a Finsler tensor field $C$ of the adjoint-type is introduced by the equation

$$
Y(f)=B^{v}(f)+Z(C(f)),
$$

where $Z(A)$, corresponding to $A \in L(n, R)$ (the Lie algebra of $G L(n, R)$ ), is a well-known fundamental vector field, defined by $Z(A)_{u}={ }_{u} T A,{ }_{u} T$ being the differential of the mapping obtained from $T$ by becoming $u \in F(M)$ fixed. $C$ as thus defined is called Cartan tensor field of $(\Gamma, N)=\left(\Gamma^{h}, \Gamma^{v}\right)$ under consideration. In the case of famous Finsler connection due to E. Cartan, $C$ is nothing but the well-known tensor, components of which are $C_{j k}^{i}$.

While the equation

$$
B^{v}(f) r=f+C(r, f), \quad C(r, f)=C(f) \gamma,
$$

will be easily verified in virtue of (1.1), the equation

$$
B^{h}(f) \gamma=D(f)
$$

introduces a new Finsler tensor field $D$ of $(1,1)$-type, which is called the deflection tensor field of $(\Gamma, N)$. It will be observed that the deflection tensor $D$ vanishes identically in the case of almost all of classical Finsler connections.

Finally, let us consider two Finsler connections $(\Gamma, N)$ and $\left(\Gamma^{\prime}, N^{\prime}\right)$, and let $B^{n}(f), B^{v}(f)$ and $B^{\prime h}(f), B^{\prime \nu}(f)$ be respective $h$ - and $v$-basic vector fields. Then, the equations

$$
\begin{aligned}
B^{\prime h}(f) & =B^{h}(f)+B^{v}\left(D^{h}(f)\right)+Z\left(A^{h}(f)\right), \\
B^{\prime v}(f) & =\quad B^{v}(f)+Z\left(A^{v}(f)\right),
\end{aligned}
$$


will be easily derived, and thus we obtain three Finsler tensor fields $D^{h}, A^{h}$ and $A^{v} ; D^{h}$ being of $(1,1)$-type, $A^{h}, A^{v}$ being of the adjoint type.

\section{§. Homogeneous Finsler connections}

Given a Finsler connection $(\Gamma, N)$, its torsions $T, C, R^{1}, P^{1}, S^{1}$ and its curvatures $R^{2}, P^{2}, S^{2}$ are introduced by the equations

$$
\begin{aligned}
{\left[B^{h}\left(f_{1}\right), B^{h}\left(f_{2}\right)\right] } & =B^{h}\left(T\left(f_{1}, f_{2}\right)\right)+B^{v}\left(R^{1}\left(f_{1}, f_{2}\right)\right) \\
& +Z\left(R^{2}\left(f_{1}, f_{2}\right)\right), \\
{\left[B^{h}\left(f_{1}\right), B^{v}\left(f_{2}\right)\right] } & =B^{h}\left(C\left(f_{1}, f_{2}\right)\right)+B^{v}\left(P^{1}\left(f_{1}, f_{2}\right)\right) \\
& +Z\left(P^{2}\left(f_{1}, f_{2}\right)\right),
\end{aligned}
$$

$C$ as appearing in (2.2) is nothing but the Cartan tensor. $S^{1}$ and $S^{2}$ are the torsion and curvature of the subordinate vertical connection $\Gamma^{v}$ respectively, and expressed by $C$ as follows.

$$
\begin{gathered}
S^{1}\left(f_{1}, f_{2}\right)=C\left(f_{1}, f_{2}\right)-C\left(f_{2}, f_{1}\right), \\
S^{2}\left(f_{1}, f_{2}\right)=\Delta^{0} C\left(f_{1}, f_{2}\right)-\Delta^{0} C\left(f_{2}, f_{1}\right)-\left[C\left(f_{1}\right), C\left(f_{2}\right)\right],
\end{gathered}
$$

where the covariant differential operator $\Delta^{0}$ is the differentiation by $Y(f)$, that is, $\Delta^{0} C\left(f_{1}, f_{2}\right)=Y\left(f_{2}\right) C\left(f_{1}\right)$.

Next, it follows from (1.1) and (2.2) that

$$
\begin{aligned}
{\left[B^{h}\left(f_{1}\right), Y\left(f_{2}\right)\right] } & =Y\left(P^{1}\left(f_{1}, f_{2}\right)\right) \\
& +Z\left(P^{2}\left(f_{1}, f_{2}\right)+\Delta^{h} C\left(f_{2}, f_{1}\right)-C\left(P^{1}\left(f_{1}, f_{2}\right)\right)\right),
\end{aligned}
$$

where the $h$-covariant differential operator $\Delta^{h}$ is the differentiation by $B^{h}(f)$, that is, $\Delta^{h} C\left(f_{2}, f_{1}\right)=B^{h}\left(f_{1}\right) C\left(f_{2}\right)$. Further, it follows from (2.4) and (1.3) that

$$
\begin{aligned}
{\left[B^{h}(f), Y(\gamma)\right] } & =B^{v}\left(P^{1}(f, \gamma)+D(f)\right) \\
& +Z\left(P^{2}(f, \gamma)+\left(\Delta^{h} C(\gamma)\right) f\right) .
\end{aligned}
$$

Now, we shall be concerned with the homogeneous property of 
some geometrical objects. A function $\mu$ on $F(M)$ is called homogeneous of degree $r$, if the equation $\mu \cdot{ }_{\alpha} H=\alpha^{\gamma} \cdot \mu$ holds good for any $\alpha \in R^{+}$. Next, a tangent vector field $X$ on $F(M)$ is called homogeneous of degree $r$, if the equation ${ }_{\alpha} H X=\alpha^{r} \cdot X$ holds good. Finally, a distribution $D$ on $F(M)$ is called homogeneous, if $D$ is $H$-invariant.

Definition 7. A Finsler connection $(\Gamma, N)$ is called homogeneous, if $\Gamma$ and $N$ be homogeneous in the respective sense of $F(M)$ and $T(M)$.

Proposition 3. A necessary and sufficient condition for $a$ Finsler connection $(\Gamma, N)$ to be homogeneous is that $B^{n}(f)$ and $B^{v}(f)$ be homogeneous of degree 0 and 1 respectively.

The proof will be easily obtained.

Proposition 4. If a function $\mu$ on $F(M)$ is homogeneous of degree $r$, then $B^{h}(f) \mu$ is homogeneous of the same degree, provided that the Finsler connection under consideration be homogeneous.

The proof will be easily obtained from Proposition 3. The following is the well-known Euler's theorem on homogeneous functions.

Proposition 5. If a function $\mu$ on $F(M)$ is homogeneous of degree $r$, then the equation $Y(\gamma) \mu=r \cdot \mu$ holds good.

Proof. Since the induced fundamental vector field $Y(f)$ is induced from the 1-parameter group of transformations $\left\{S_{t f}\right\}$, it is seen that, at a point $u=(y, z)$,

$$
\begin{aligned}
Y(\gamma)_{u} \mu & =\lim _{t \rightarrow 0} \frac{1}{t}\{\mu(y+t y, z)-\mu(y, z)\} \\
& =\lim _{t \rightarrow 0} \frac{1}{t}\{\mu \cdot(1+t) H(u)-\mu(u)\}
\end{aligned}
$$




$$
=\lim _{t \rightarrow 0} \frac{1}{t}\left\{(1+t)^{r} \mu(u)-\mu(u)\right\}=r \cdot \mu(u) .
$$

The following theorem gives the interesting properties of a homogeneous Finsler connection, although (2) will not need in future.

Theorem 3. The torsion $P^{1}$ and the curvature $P^{2}$ of $a$ homogeneous Finsler connection satisfy

(1) $P^{1}(f, \gamma)=-D(f)$,

(2) $P^{2}(f, \gamma)=-\Delta^{h} C(\gamma, f)-C(D(f))$.

Proof. We first obtain from (2.4) one of the Ricci's identities

$$
\begin{gathered}
\Delta^{h}\left(\Delta^{0} K\right)\left(f_{2}, f_{1}\right)-\Delta\left({ }^{0} \Delta^{h} K\right)\left(f_{1}, f_{2}\right) \\
=\Delta^{0} K\left(P^{1}\left(f_{1}, f_{2}\right)\right)-P^{2}\left(f_{1}, f_{2}\right) K-\left(\Delta^{h} C\left(f_{2}, f_{1}\right)\right) K+C\left(P^{1}\left(f_{1}, f_{2}\right)\right) K
\end{gathered}
$$

If we put $f_{1}=f$ and $f_{2}=\gamma$ in the above, it follows that

$$
\begin{aligned}
\Delta^{0} K & \left(P^{1}(f, \gamma)\right)-P^{2}(f, \gamma) K-\left(\Delta^{h} C(\gamma, f)\right) K+C\left(P^{1}(f, \gamma)\right) K \\
& =\Delta^{h}\left(\Delta^{0} K\right)(\gamma, f)-\Delta^{0}\left(\Delta^{h} K\right)(f, \gamma) \\
& =\Delta^{h}\left(\Delta^{0} K(\gamma)\right)(f)-\Delta^{0} K\left(\Delta^{h} \gamma(f)\right)-\Delta^{0}\left(\Delta^{h} K(f)\right)(\gamma) .
\end{aligned}
$$

If $K$ is supposed to be homogeneous of degree 1 , it follows from Propositions 4 and 5 that

$$
\Delta^{h}\left(\Delta^{0} K(\gamma)\right)(f)=\left(\Delta^{h} K\right)(f), \quad \Delta^{0}\left(\Delta^{h} K(f)\right)(\gamma)=\Delta^{0} K(f),
$$

and hence the above equation leads us to

$$
\begin{aligned}
\Delta^{0} K\left(P^{1}(f, \gamma)\right. & +D(f))-\left(\Delta^{h} C(\gamma, f)\right) K+C\left(P^{1}(f, \gamma)\right) K \\
& =P^{2}(f, \gamma) K .
\end{aligned}
$$

Therefore, the equation (1), together with the above equation, gives (2). In order to prove (1), it is sufficient to show that $\left[B^{h}(f)\right.$, $Y(\gamma)]$ is vertical, because of (2.5). Let $\mu$ be any homogeneous function of degree 1 on $T(M)$, and then $\mu \cdot \pi_{1}$ is obviously homogeneous of degree 1 on $F(M)$. It then follows from Propositions 4 and 5 that 


$$
\begin{gathered}
\left(\pi_{1}\left[B^{h}(f), Y(\gamma)\right]\right) \mu \\
=B^{h}(f)\left(Y(\gamma)\left(\mu \cdot \pi_{1}\right)\right)-Y(\gamma)\left(B^{h}(f)\left(\mu \cdot \pi_{1}\right)\right)=0,
\end{gathered}
$$

from which the equation $\pi_{1}\left[B^{n}(f), Y(\gamma)\right]=0$ is derived.

Definition 8. The D-simple Finsler connection $\left(\Gamma^{\prime}, N^{\prime}\right)$ of a Finsler connection $(\Gamma, N)$ is defined by (1.4) and (1.5), where $D^{h}$ $=A^{v}=0$ and $A^{h}(f)=-P^{1}(f, \quad)$.

The following proposition will be immediately shown from (1.3), (1.4) and (1.5).

Proposition 6. The D-simple Finsler connection $\left(\Gamma^{\prime}, N^{\prime}\right)$ of a Finsler connection $(\Gamma, N)$ is such that
(1) $N^{\prime}=N$,
(2) $D^{\prime}(f)=D(f)+P^{1}(f, \quad)$.

Theorem 4. The deflection tensor $D^{\prime}$ of the D-simple Finsler connection $\left(\Gamma^{\prime}, N\right)$ of any homogeneous Finsler connection $(\Gamma, N)$ vanishes identically.

This important theorem is a direct result of Theorem 3-(1) and Proposition 6-(2).

\section{§3. Homogeneous non-linear connections}

First of all, we shall consider the differential of the characteristic field $\gamma$, the mapping $F(M) \rightarrow F,\left[(y, z) \rightarrow z^{-1} y\right]$.

Proposition 7. The differential of the characteristic field $r$ is given by

$$
{ }_{2} a \cdot r X=\pi_{1} X-a_{f} \cdot \pi_{2} X,
$$

where $X \in F(M)$ " and $u=i^{-1}(z, f)$.

Proof. It follows from the identification $i: F(M) \rightarrow L(M) \times F$ and the mapping $a: L(M) \times F \rightarrow T(M)$ that

$$
\pi_{1} X=a \cdot i X=a\left(\pi_{2} X, \gamma X\right)=a_{f} \cdot \pi_{2} X+{ }_{2} a \cdot \gamma X,
$$


which proves the proposition.

Let us remember the definition of a Finsler $\operatorname{triad}\left(\Gamma_{F}, N, \Gamma^{v}\right)$, where there is not any interrelationship among $\Gamma_{F}, N$ and $\Gamma^{v}$. Now, a special Finsler triad is required for our purpose.

Definition 9. A Finsler connection $(\Gamma, N)$ is called $N$-simple, if $N$ is the associated non-linear connection with the subordinate $F$-connection $\Gamma_{F}$.

A geometrical meaning of the deflection tensor $D$ will be given by the following.

Theorem 5. A necessary and sufficient condition for a Finsler connection to be $N$-simple is that the deflection tensor $D$ vanishes identically.

Proof. It follows from (1.3) that $r B^{n}(f)=P(D(f))$, and hence Proposition 7 leads us to

$$
{ }_{2} a P\left(D\left(f_{1}\right)\right)=\pi_{1} B^{h}\left(f_{1}\right)-a_{f} \cdot \pi_{2} B^{h}\left(f_{1}\right),
$$

for any $f_{1} \in F$. The proof follows then immediately from the definition of the subordinate $F$-connection.

The main result of the present paper is now stated as the following theorem on a homogeneous non-linear connection.

Theorem 6. Any homogeneous non-linear connection in the tangent bundle $T(M)$ is the associated one with an F-connection in the bundle of linear frames $L(M)$.

Proof. Let $N$ be a given homogeneous non-linear connection in $T(M)$, and construct a homogeneous Finsler connection $(\Gamma, N)$, combining with $N$ an arbitrary homogeneous connection $\Gamma$ in $F(M)$. In order to do so, it is enough to observe that the induced connection $\Gamma$ from a linear connection $\underline{\Gamma}$ in $L(M)$ by the induced mapping $\pi_{2}$ is surely homogeneous, where $\Gamma$ is given by 


$$
\Gamma_{u}=\left\{X \in F(M)_{u} \mid \pi_{2} X \in \underline{\Gamma}_{z}, z=\pi_{2} u\right\} .
$$

Next, let us construct the $D$-simple Finsler connection $\left(\Gamma^{\prime}, N^{\prime}\right)$ of the above $(\Gamma, N)$. It then follows from Proposition 6 that $N^{\prime}=N$, and from Theorem 4 that $D^{\prime}=0$. Therefore, Theorem 5 leads us to the conclusion that the connection $\left(\Gamma^{\prime}, N^{\prime}\right)$ is $N$-simple, that is, the original non-linear connection $N$ is the associated one with the subordinate $F$-connection $\Gamma_{F}^{\prime}$ of $\left(\Gamma^{\prime}, N^{\prime}\right)$.

It should be remarked that a homogeneous non-linear connection $N$ may be associated with two different $F$-connections. It is, however, observed that the above $F$-connection $\Gamma_{F}^{\prime}$ satisfies $\Gamma_{(\alpha f)}^{\prime}=\Gamma_{(f)}^{\prime}$ for any $\alpha \in R^{+}$. In general, we have

Proposition 8. The subordinate F-connection $\Gamma_{F}=\left\{\Gamma_{(f)}\right\}$ of $a$ homogeneous Finsler connection $(\Gamma, N)$ satisfies $\Gamma_{(\alpha f)}=\Gamma_{(f)}$ for any $f \in F$ and any $\alpha \in R^{+}$.

Proof. The distribution $\Gamma_{(\alpha f)}$ is defined by $\Gamma_{(\alpha f) z}=\pi_{2} \Gamma_{u^{\prime}}^{h}$, where

$$
u^{\prime}=i^{-1}(z, \alpha f)=(z \alpha f, z)={ }_{\alpha} H(z f, z)=\alpha u, \quad u=i^{-1}(z, f) .
$$

Therefore we see

$$
\Gamma_{(\alpha f) z}=\pi_{2} \Gamma_{\alpha u}^{h}=\pi_{2} \cdot{ }_{\alpha} H \Gamma_{u}^{h}=\pi_{2} \Gamma_{u}^{h}=\Gamma_{(f) z} .
$$

\section{References}

[1] Barthel, W.: Nichtlineare Zusammenhänge und deren Holonomie-gruppen. J. reine angew. Math. 212, 120-149, 1963.

[2] Matsumoto, M.: Affine transformations of Finsler spaces. J. Math. Kyoto Univ. 3 , 1-35, 1963.

[3] Matsumoto, M.: Linear transformations of Finsler connections. J. Math. Kyoto Univ. 3, 145-167, 1964.

[4] Matsumoto, M.: Paths in a Finsler space. J. Math. Kyoto Univ. 3, 305-318, 1964.

[5] Matsumoto, M.: On R. Sulanke's method deriving H. Rund's connection in a Finsler space. J. Math. Kyoto Univ. 4, 355-368, 1965.

[6] Matsumoto, M.: A Finsler connection with many torsions. Tensor (N. S.) 17, 217-226, 1966.

[7] Matsumoto, M.: Connections, metrics and almost complex structures of tangent bundles. J. Math. Kyoto Univ. 5, 251-278, 1966.

[8] Matsumoto, M.: Theory of Finsler spaces and differential geometry of tan- 
gent bundles. J. Math. Kyoto Univ. 7, 169-204, 1967.

[9] Nomizu, K.: Lie groups and differential geometry. Publ. Math. Soc. Japan 2, 1956.

Institute of Mathematics, Yoshida College, Kyoto University 